\title{
Family Therapy and Childhood-Onset Schizophrenia: Pursuing Clinical and Bio/Psycho/Social Competence
}

Stanley D. Hall

Brigham Young University - Provo

Roy A. Bean

Brigham Young University - Provo, roy_bean@byu.edu

Follow this and additional works at: https://scholarsarchive.byu.edu/facpub

Part of the Other Social and Behavioral Sciences Commons

\section{Original Publication Citation}

*Hall. S. D., \& Bean, R. A. (2008). Family therapy and childhood-onset schizophrenia: Pursuing clinical and bio/psycho/social competence. Contemporary Family Therapy, 30, 61-74.

\section{BYU ScholarsArchive Citation}

Hall, Stanley D. and Bean, Roy A., "Family Therapy and Childhood-Onset Schizophrenia: Pursuing Clinical and Bio/Psycho/Social Competence" (2008). Faculty Publications. 5052.

https://scholarsarchive.byu.edu/facpub/5052 


\title{
Family Therapy and Childhood-Onset Schizophrenia: Pursuing Clinical and Bio/Psycho/Social Competence
}

\author{
Stanley D. Hall · Roy A. Bean
}

Published online: 4 March 2008

(C) Springer Science+Business Media, LLC 2008

\begin{abstract}
Childhood-onset schizophrenia is perceived as more complicated because of its complexity, pervasive duration, and impact on individual and family functioning. Viewing the symptoms of childhood schizophrenia as creating a bio/psycho/social competence within which the therapist and family must interact may help contribute to treatment success. Competent family therapists working within this culture should be knowledgeable about the characteristics of childhood-onset schizophrenia, aware of their own biases towards psychosis, and skillful in its treatment. Guidelines are provided to help therapists identify their attitudes about schizophrenia and understand appropriate skills for treating the disorder.
\end{abstract}

Keywords Family therapy · Childhood-onset schizophrenia - Therapist competence

\section{Introduction}

Illness and disease, whether physical or mental, have the potential to be all-consuming and may dominate multiple dimensions of a client system's daily experience. Morris (2000) suggests that medical illnesses create a bio-culture that envelopes patients and their families, exposing them to the worlds of hospitals, medicine, doctors and other treatment providers. Simultaneously, affected families must negotiate any differences between their own beliefs about the illness and the views held by society. In accordance with a biocultural perspective, it is theorized that mind and body affect one another in a bi-directional fashion while a similar reciprocal causal association can be noted in the relationship between the affected client system and the larger society. This phenomenon can be seen, easily and dramatically, in the experience of those who suffer with HIV infection or AIDS, due to the numerous medical, social, financial and relational aspects of the illness, and the stigma imposed by society on those suffering with the illness (Brown et al. 2003).

S. D. Hall · R. A. Bean $(\square)$

Marriage and Family Therapy Program, School of Family Life, Brigham Young University, 258 TLRB, Provo, UT 84602, USA

e-mail: roy_bean@byu.edu 
By extension, there is a bio-culture, or more appropriately, a bio/psycho/social culture associated with a variety of mental illnesses and relational problems. Some disorders are, in particular and as a function of their characteristics, more likely to create a set of cultural practices and rituals dictating such things as appropriate communication among family members, acceptable behaviors, and client responses to other systems. One such disorder with a very high likelihood of disrupting and reshaping individual and family functioning is childhood-onset schizophrenia.

With the onset of schizophrenia, patterns of individual and family functioning often undergo a dramatic change and considerable time and energy are spent in adjusting to associated symptoms. For example, siblings may not feel comfortable bringing friends to the house if a mentally ill brother or sister is there, and they generally have to reorganize much of their lives to accommodate the difficult circumstances imposed by the schizophrenia (Gerace et al. 1993). Family interactions are changed and impacted in many ways while parents are challenged with their child's violence, withdrawal and verbal aggression (Ferriter and Huband 2003). In addition, parents usually have to make significant changes in their work schedules and finances to accommodate treatment plans and hospitalizations.

In many instances, schizophrenia forces families to live in a world that is distinctly different from what others understand and experience because of the interruptive psychological and interpersonal symptoms and the requisite mental health care system they must navigate. Thus, it is not inappropriate to suggest that schizophrenia creates a culture that is largely inseparable from the illness, one that the individual and family must deal with on a daily basis. Viewing the collective experiences of the client system as a culture may not be fully accurate. However, this conceptualization is beneficial when it allows therapists and family members to work toward greater "cultural competence" in working with the family's collective experience with schizophrenia.

Informed by one of the most prominent theoretical models (Sue et al. 1992; Sue and Sue 2003), there are three primary dimensions utilized in training and evaluating culturally competent therapists and counselors: therapist knowledge, therapist awareness, and therapist skills. A lack of competence in any of these three dimensions will put the therapist at a disadvantage and may even be deleterious to the family. In order to illustrate more fully the benefits of applying this model of therapist cultural competence to the topic of schizophrenia, a detailed discussion of each dimension is provided below along with important applications for therapists.

\section{Becoming More Culturally Competent}

For schizophrenia, comorbidity with other mental health illnesses is often high, especially in the case of substance abuse (Mueser et al. 2006), depression (Drake et al. 2004), and neurotic syndromes, emotional disorders, and conduct disorders (Hafner and Nowotny 1995). Autism and mental retardation also have been found to be co-morbid with schizophrenia, especially in the case of early-onset schizophrenia (Fagerlund et al. 2006). In instances where individuals are dually diagnosed with early onset schizophrenia and autism/mental retardation, long-term hospitalization or even more intensive services are often required. Consequently, as a qualifier, this article focuses primarily on early-onset schizophrenia with individuals who do not have these Diagnostic and Statistical Manual of Mental Disorders-IV (DSM-IV-TR, American Psychological Association [APA] 2000) axis II disorders because of their more complex needs. 
Therapist Knowledge

In order to be considered competent when working with families imbedded in the bio/ psycho/social culture of schizophrenia, it is important that therapists and counselors have knowledge regarding various topics. The information presented here is considered to be most related to a basic, competence-level knowledge of schizophrenia and its properties.

\section{General Characteristics}

Schizophrenia is generally considered a disorder of exclusion, with disorganization of thought, speech, and behavior being universal symptoms (APA 2000). In recent years, schizophrenia research has revealed a deterioration of the brain (Gogtay et al. 2004; Sowell et al. 2000) leading to disorganization of thought, speech, and behavior as it affects the central nervous system (Hata et al. 2003). More rarely, rigidity in the body or catatonia also can be present (Cantor 1988).

Psychotic symptomotology, including delusions and hallucinations, often define the illness, and can occur in the case of children as well (Gur and Johnson 2006). Delusions are defined as unchangeable false beliefs held by the patient, whether they are believable or bizarre, while hallucinations are any unverifiable extra-sensory perceptions the patient experiences, including auditory voices (inside or outside of the head), visual hallucinations, or other sensations (APA 2000). Delusions, hallucinations, and catatonia are referred to as positive symptoms, and their early resolution can help alleviate the depression that often accompanies these symptoms (Drake et al. 2004). It is considered to be very important to attend to symptoms of psychosis because of the high risk of suicidality among juveniles (Schwartz-Stav et al. 2006).

Negative symptoms are characteristics absent from a patient's behavior, speech, attitude, thinking, or volition. These tend to be more persistent and difficult to alleviate than the cyclical positive symptoms that appear and disappear in stages. Negative symptoms may include a loss of desire to participate in activities, lack of motivation or volition, loss of speech, flattening of emotions, disorganization, and difficulty expressing feelings.

\section{Early Onset Schizophrenia}

The diagnosis of early onset schizophrenia (under age 14) is rare. However, in part, this may be a function of the symptoms being ignored or going unrecognized. Therapists and parents attuned to the subtle presentation of early symptoms may be able to identify schizophrenia in its prodromal stages when active psychosis has not yet manifested itself (Gur and Johnson 2006). In fact, research suggests that well-crafted and sensitive interviewing techniques are effective in identifying whether or not a child has schizophrenia or is in its beginning stages (Asarnow 1994).

The specific manifestations of schizophrenia appear to be influenced by a number of contextual factors, including the child's level of cognitive development. Children have more limited associations in their mind with which to create delusions and so they are generally simplistic in comparison to adult delusions. As such, delusions may not appear as dramatic or outlandish as those typically associated with adult-onset schizophrenia, and, in some cases, may be easily confused with the fantastical, imaginary thinking typical of young children. As a further complicating factor, many of the early onset symptoms can be considered overlapping with other childhood mental illnesses. An accurate assessment 
among teens is similarly complicated due to the challenge of separating the disorder's initial manifestation from behaviors often viewed as normative among Western adolescents. For example, when affected teens have hallucinations, parents and clinicians may conclude that symptoms are drug-related due to the widely-held belief that disturbed adolescents are prone to drug experimentation. Consequently, assessment and diagnosis of early onset schizophrenia can be viewed as more difficult and nuanced in comparison to the late or adult onset version.

Overall, childhood-onset schizophrenia has a more severe prognosis than adult-onset, with more negative symptoms (Yang et al. 1995), and is usually more pervasive and insidious (Delbello and Grcevich 2004). The chance for remission is approximately half the expected rate for adults, and diagnosis is generally stable until at least adulthood (Maziade et al. 1996). Comorbidity with other disorders is high, including a large percentage of youth who experience dysthymia, conduct disorder, or oppositional defiant disorder (Russell et al. 1989). Considering these associated problems, it is even more important that children and their families receive early interventions to offset the course of the illness and to allow for more normal development, less significant psychotic episodes, and the possibility of complete or partial remission (Crim and Fitzpatrick 2003). Nevertheless, the stigma associated with schizophrenia and the chance of error cause many therapists to hesitate before diagnosing children even when symptoms are present.

\section{Systemic Effects of Schizophrenia}

Given that even unaffected children require considerable attention and care, the added burden of managing schizophrenia-related problems and demands can be overwhelming for parents and caregivers. Parents must face a number of difficult sentiments including: confusion about what to do, feelings of inadequacy, a fear of being too optimistic about the child's functioning, and a loss of self because of the necessary focus on the child (Chaffey and Fossey 2004). Parents also have to develop new skills and learn new tasks as they care for their child, and other areas of their life (e.g., work, social life, marriage) can become secondary to caring for the diagnosed individual.

McClellan et al. (1993) identified a high rate of disruption in the families they studied that included a child with schizophrenia. Children in this study who were diagnosed with schizophrenia or a similar disorder were found to have significant deterioration in outcomes when compared to the children suffering from a mood disorder. A tremendous amount of energy and productivity are lost in families when a child has schizophrenia, leading to unexpected hidden costs, and high emotional burdens that all family members feel, particularly the parents, (Werry 1996). Siblings of the symptomatic individual may suffer feelings of loss and sadness. Anger and resentment also can be experienced by family members in terms of a demand to "snap out of it" when symptoms begin impacting the affected child's interpersonal relationships, academics, work, and personal self-care and grooming.

\section{Medications and Illegal Drugs}

Antipsychotic medications used for adults are used for children but may have varying results, so it is important for physicians to closely monitor clinical manifestations of psychosis (Delbello and Grcevich 2004). While relatively little psycho-pharmacological research has been conducted focusing on schizophrenia in children, there is some evidence that antipsychotics can have special benefits for youth (Dulmus and Smyth 2000). In fact, Armenteros and Davies (2006) initiated an evidence-informed baseline for medications 
used to treat psychosis in children, allowing psychiatrists to prescribe more appropriate dosages with expected results.

While not within the scope of practice for therapists to make suggestions to clients regarding medications, clinicians can be helpful in monitoring medication-related benefits and side-effects. In this respect, they act as a part of a cross-disciplinary treatment team that is usually required to manage this disorder effectively and, as needed, can act as an "interpreter" for the family members and their medical care providers.

Consistent with the knowledge dimension of therapist cultural competence, it is important for the therapist to be familiar with antipsychotic medications in general. The first antipsychotic medications that were created are now called typicals (e.g., thorazine) and continue to be about as effective as the more recent atypicals (e.g., risperdal, clozapine). The primary difference is that the majority of the atypical medications used today lack many of the extra-pyramidal (neurological) symptoms that can create awkward involuntary body movements, or jerkiness. Clozaril, the first atypical on the market, is also one of the most potent medications. However, its potentially harmful side-effects to the immune system have caused it to be reserved as a last resort by most psychiatrists. Medications can be administered in either pill or injection form, with long-lasting injections prescribed for those not willing to maintain compliance with standard psychopharmacological protocol.

In the case of illegal and/or prescribed drugs, abuse can lead to hallucinogenic symptoms, which can interfere with the assessment of schizophrenia. For example, it is difficult to make a straight-forward determination about whether a youth is isolating and irritable because of schizophrenia, or if it is related to his or her drug use and accompanying evasive behaviors. Juveniles taking illicit drugs significantly increase their risk of developing a major mental illness and many cases of childhood-onset schizophrenia were preceded by or developed simultaneously with the use of illegal drugs (Veen et al. 2004). In addition, even after a clear diagnosis, drug use represents a complicating factor as it can interfere with the efficacy of prescribed medications and/or prevent compliance with treatment.

\section{Therapist Awareness}

In order to facilitate greater levels of cultural competence, it is necessary for therapists to be aware of their own assumptions, values, and biases. More specifically, therapists should be aware of any negative emotional reactions they may have towards those with serious mental illness. This is particularly important as individuals with serious mental illness may discern a therapist's stereotypes and preconceived notions, or may be hyper-sensitive to any seemingly judgmental interaction. Gaining a greater awareness of one's own beliefs can help therapists identify professional limits and accept differences between themselves and their clients (Sue et al. 1992). In order to more fully illustrate this dimension of therapist competence applied to the systemic culture of schizophrenia, several main awareness-related issues are presented below.

\section{Mental Illness does not Equal Violence}

Therapists should be aware of how the media often sensationalizes any violence committed by someone with a mental health issue, especially if the person was psychotic at the time of violent act. Overall contributions to crime in society by individuals with schizophrenia are relatively small and certainly not a rule. Assuming that a client is violent without supportive evidence can hinder the formation of a healthy alliance, which has been shown to be crucial to recovery (Topor et al. 2006). 


\section{Caution in Avoiding Stigmatization}

Health providers are not exempt from stigmatizing attitudes and still may discriminate in subtle ways (e.g., minimizing client emotional needs when client is hallucinating), which can lead to isolating behavior on the part of the client (Chin and Baton 2006). Many individuals with schizophrenia lack insight into their own illness and may not be aware of how they are different from others. However, clients with greater awareness of their illness tend to be sensitive to even subtle shaming or stigmatizing looks from others (Pompili et al. 2004). In fact, an awareness of others' opinions about them (real or imagined) appears to be related to suicide attempts and to depression in clients (Schwartz-Stav et al. 2006). As a result, therapists need to continually evaluate their own behaviors and interactions in order to avoid contributing to stigma and any associated risks.

Therapist self-monitoring is also essential in preventing already sensitive and even paranoid clients from accusing the therapist of being fearful or judgmental. Parents also may be hypersensitive to judgment and concerned about how they and their child are judged by neighbors, teachers, friends, and others. To prevent misunderstandings and difficulties in the formation of therapeutic alliances, therapists should strive from the beginning to create a safe place for all members of the client family.

\section{Separating the Client System from the Illness}

Therapists should be aware of their own feelings towards those with serious mental illness and evaluate whether they can view the person outside of the illness. If not, they will not be able to work with that person and the family in a way that supports their right to respect and dignity. One simple adjustment that can be made is in relation to one's clinical vocabulary, because referring to the youth as a schizophrenic can be seen as dehumanizing and impersonal. Children should be helped to see themselves outside of the illness and as having numerous qualities separate and distinct from the illness.

Another of the clinician's primary tasks is to help the family externalize the disorder and identify other important matters in their life that should not be neglected. In doing so, it is important to avoid the use of pejorative systemic labels that unfairly attribute mental illness causation to the family. In this regard, therapists should be aware that traditional psychotherapy terminology referring to the genesis of schizophrenia and the double-bind theory, including phrases like "schizophrenogenic mother" and "schizophrenogenesis" are inappropriate, blaming, and generally counterproductive to treatment.

\section{Avoiding a Biased Stance}

Given the "unfair burden" of schizophrenia-related symptoms that children may suffer, therapists may be particularly sympathetic to children's needs and contribute to their adversarial position relative to the rest of their family. Therapists need to be aware of the possibility of inadvertently condemning the family for poor communication, and even blaming them for the child's symptomatology. This can be particularly challenging given that research findings suggest a relationship between high levels of expressed emotion (framed as hostility or conflict) and relapses in positive symptoms (Butzlaff and Hooley 1998; Ng et al. 2001).

Alternatively, it is relatively easy to adopt a biased stance against the affected child because family members often present as embattled, beleaguered and weary from months 
or years of dealing with symptoms. Therapists who are aware of this systemic "tug-ofwar" can avoid colluding with a single family member or subsystem, and will have more success in developing and maintaining the clients' trust in the therapeutic relationship.

\section{These Families are Resilient}

While a sympathetic understanding of the family's burden is important to maintain, therapists should be wary of viewing family members as fragile. They would not have been able to survive, either as individuals or as a family unit, without a great deal of hard work, patience, flexibility, and other psychosocial strengths. In this regard, it is important for clinicians to avoid treating them as helpless victims or over-protecting them from clinical challenges and familial responsibilities. Families will differ in levels of overall resilience based on availability of resources (e.g., economic, spiritual, social (Patterson 2002)) and their levels of individual and collective resilience certainly will vary over the course of treatment. However, it is beneficial to view the family as suffering the problem together and then working to help them improve together, as the resilience of the individual in crisis is usually associated with the resilience of the entire family (Patterson 2002).

\section{Practicing Appropriate Self-Care}

Some therapist struggle with a type of "savior-complex," believing that they must save the client at any cost (Gartner 1985). This is a significant concern when working with seriously mentally ill clients because they require considerable attention, and burnout is a genuine risk for therapists dealing with schizophrenia. Therapists need to be aware of their own level of emotional fatigue as they join the family system in working with an exhaustive and on-going set of individual and family symptoms. Additionally, while it is easy to feel overwhelmed by the complexity and pervasive nature of the disorder, it is important for clinicians to practice appropriate self-care. In so doing, they model this practice for the affected family members and help the family be prepared for the long-term process of coping with issues at a reasonable pace.

\section{Avoiding Personalizing Client Delusions}

The DSM-IV-TR (APA 2000) excludes all religious doctrine from being pathologized, yet client delusions often have strong religious overtones. While it is important to realize that children raised in religious families may manifest particular doctrines as central themes in their hallucinations and delusions, research suggests that there is no difference in severity of religious delusions between groups of irreligious and religious individuals with schizophrenia (Getz et al. 2001). Being aware of this will help the therapist avoid any inclination to pathologize a family's religiosity or discourage involvement in a church community. Regardless of religious activity, parents are expected to be concerned when children profess to be, or to receive communications from, Jesus Christ or some other religious/supernatural being. Parents and therapists should not experience these delusions as offensive or blasphemous and should, in most cases, avoid attempts to challenge these fixed delusions. Otherwise, they increase the risk of the affected child responding with more angry outbursts or a cessation of sharing said delusions. 


\section{Minimizing Child Symptoms}

As another potential awareness issue, there may be therapists who see an undiagnosed child's bizarre behavior or delusion as a sign of great creativity and imagination, and hesitate in referring the child for appropriate assessment or treatment. While this reframe might be helpful in some instances, it also may prevent the child from receiving the services he/she requires for treatment. Systems-based therapists often have conflicted feelings toward the medical model, and the associated DSM-IV-TR (APA 2000) categorization of individuals, so it may be particularly important for marriage and family therapists (MFTs) to examine their own anxiety about diagnosing children or adolescents with serious mental illness and referring them for a deeper psychiatric examination. Clearly, the risks of ignoring schizophrenia are considerable and include suicide, violence, and other severe symptom-related problems.

\section{Fear of Being Ineffective}

Schizophrenia is among the most prominent illnesses in psychiatric hospitals and relapse is common (Prince 2006), which may lead therapists to see cases of schizophrenia as hopeless. In dealing with this possible sentiment, it is recommended that therapists consider several important points. First, if the therapist is feeling a degree of hopelessness and frustration, it is very likely that the family experiences these feelings even more profoundly. Second, while complete recovery is very unlikely, some greater degree of productivity and functioning is possible. In this regard, it is important to remember (and to remind the family) of where they are currently as compared to the baseline of the "worst it has ever been." Finally, therapists need to appreciate that the changes may be most evident in the lives of parents and other family members rather than in the diagnosed individual. In this regard, systems-trained therapists are extremely well-positioned to help the family system and all its members survive and even thrive.

Professional and insurance-driven demands for quick, effective treatments also may create a level of frustration for some therapists who are trying to be brief in their treatment approach. Being aware of this agenda and considering one's own impatience as a healer will help therapy meet the needs of the family rather than the therapist's own need to "close the file" on another successful case. Instead, a therapist usually will need to identify small, progressive steps in the process of alleviating symptoms, and focus on bringing about small degrees of stability and change in individual and family functioning.

Clearly, there is ample room for therapist self-doubt when considering whether one is actually qualified to treat schizophrenia, prompting many therapists to view psychiatrists as those primarily responsible for treatment. Self-evaluation regarding one's scope of practice is appropriate and ethical. However, in actuality, many psychiatrists want MFTs to help manage the treatment of family-related issues (Crim and Fitzpatrick 2003). Consequently, it is important for MFTs to recognize any legitimate need for additional, specialized training in working with schizophrenia while at the same time realizing that this disorder can cause very skilled clinicians to question their competence and effectiveness.

\section{Therapist Skills}

A discussion of the necessary skills for therapists working within the bio/psycho/social culture imposed by schizophrenia is best presented referring to: (a) the multi-faceted role 
that they must play and (b) the applicable treatment models for this disorder. In reference to the first category, clinicians carry out a variety of roles including that of assessor, grief counselor, team player, facilitator/educator, and interventionist. Each of these roles is discussed in terms of duties and the associated skills.

\section{Assessor}

Assessment is a critical role for the therapist and should be applied throughout treatment, although it is most critical in the early stages of the illness. Assessing for recreational drug use and the frequency of use can provide therapists with a baseline of whether the behaviors occur only when the youth is using drugs. Whether a youth is isolating and irritable because he or she is not fitting into school socially, or whether it is because he or she is demonstrating negative symptoms of schizophrenia requires close observation and verbal assessment. Assessing for potentially violent delusions and helping the client find safe resolutions will help alleviate the therapist's and family's fears of violent acts from the client. Additionally, effective evaluations can prevent hospitalizations if early assessments indicate a referral for medication consultation to decrease violent tendencies (Gilligan and Lee 2004). In making a refined and accurate assessment, it is also important for therapists to consider the impact of ethnic culture and acculturation level. For example, this is relevant in terms of whether disorganized speech might be a function of speaking English as a second language or where a culture's emphasis on spirituality and communication with deceased ancestors may suggest distorted thinking or hallucinations.

\section{Grief Counselor}

Therapists often will need to help the family cope with feelings of loss, depression, and anxiety. Confusion and sadness are natural responses from parents when they see the child they knew so well change in such a dramatic fashion. Being familiar with grief work will help the therapist be creative in helping the family to adjust to this ambiguous loss when there have been psychological losses, yet the physical person remains (Boss et al. 1988; Boss and Couden 2002). Hopes for the future held by the parents and siblings may be lost or seriously impeded with the diagnosis of a serious illness in a child (Marsh and Johnson 1997). Furthermore, parents may feel consumed by the treatment of this child, much as cancer patients feel consumed by the grief and loss of their illness (Lev and McCorkle 1998). Experiences of grief and doubt about the future should be listened to carefully and identified as unique. This will help to assuage the pain and assist the family to understand the ambiguity of their losses, thereby creating hope and the possibility for creative solutions (Boss and Couden 2002). Clinicians should work to encourage parents in developing and maintaining the courage to face the future even when it seems fraught with obstacles and potential disappointments (Hartshorne 2002).

\section{Team Player}

Therapists may find it beneficial to work as part of a larger team that is already dealing with psychosis in children. Specific training and experience in working with this population of youth will make one more confident in the work and the client will be benefited by the added knowledge and expertise. Unfortunately, many MFTs do not feel confident in treating schizophrenia (Northey and Harrington 2003). Consulting with other professionals 
and teaming up with a psychiatrist who already may be prescribing medication to the youth will provide support for the therapist. Psychiatrists and others who have received more training in understanding severe mental illness can be resources for practical advice in working within the culture of schizophrenia. While many psychiatrists are capable of managing aspects of early-onset schizophrenia (Shaw and Singh 2004), most are aware of the limitations on their time and/or their lack of training in systemic practice.

In the majority of instances, clinicians should encourage familial participation throughout therapy because of the significant and even dramatic influence that family members can have in the child's life (Wudarsky et al. 2001). In this way the therapist should be able to identify and resolve previously unmet family needs, test for treatment fit, and address communication and behavior issues in the family (Lewis et al. 2005). On occasion, it will be beneficial to speak with family members without the identified patient present, especially when discussing topics that are possible triggers to the child's delusions. More commonly, the parents will need additional support and training and, in these instances, it will be easier to attend to the parental/caregiver subsystem without the affected child present.

\section{Facilitator/Educator}

In working toward the goal of greater stability, a therapist's work will be only one component of the treatment necessary for the family and the diagnosed individual. Social, academic, or vocational rehabilitation may be the primary focal points for the individual when he or she comes to therapy. In this role, therapists help facilitate connections to other aspects of the helping services by (a) being aware of pharmacological treatments/sideeffects and referring to psychiatric care; and (b) connecting affected youth with vocational rehabilitation centers or academic support programs.

Hospitalization and other treatments can be very costly and may be a significant stressor for the family. By being aware of federal grants and programs, therapists can help the family access financial resources and support. Depending on state and community funding for mental health initiatives, local case managers may be available to identify available services and help with the individual needs of the affected child while family therapy continues for the caretakers and larger family system.

Acting as an educator for the family can be crucial in helping them accept the changes they are seeing in their child and how it may affect their future. Relapse can come on suddenly and shock family members, creating no small division between members of the family in identifying options and trying to settle on the best treatment. A referral to the National Association of Mental Illness (NAMI) also can be helpful to families in normalizing their emotions and identifying other families who are suffering from similar issues.

\section{Interventionist}

Acting as an interventionist is consistent with the training of most MFTs. However, there are a few particular aspects of this role that need further elaboration. First, clinicians should work with the child in such a way that he/she feels free to share whatever may be bothering him or her. Even when a delusion appears unbelievable or bizarre, the therapist should remain nonjudgmental and noncondemning. Instead, a therapist should be empathetic toward the child and acknowledge his or her experiences as real without encouraging or confirming his or her beliefs. 
Another primary task is helping both the caregivers and the child externalize the disorder and prioritize important matters in their life. This can be modeled by the therapist's own language and discussion, identifying the illness as an important part of their life, but one that is still only a part of their life. Children should be helped to see themselves as separate from schizophrenia, so as to not base their identity solely on the illness. Speaking to the client and his or her family as equals and avoiding potentially offensive or stigmatizing language will facilitate greater sensitivity to the family's struggles and needs.

\section{Applicable Models of Intervention}

While relatively little research has been conducted focusing on childhood-onset schizophrenia, some treatment guidelines and therapist skills can be inferred from what is available. One area that has received considerable research attention is the topic of high expressed emotion, although the majority of the empirical studies have focused on adultonset schizophrenia. High expressed emotion (HEE) is defined as an exaggeration of criticism, hostility, intrusiveness, and over involvement in the relationship, and has been found to exacerbate schizophrenia-related symptoms and provoke relapse (e.g., $\mathrm{Ng}$ et al. 2001; Butzlaff and Hooley 1998).

Relative to the area of therapist skills, Kuipers (2006) found that lowering high levels of expressed emotion in affected families appears to prevent relapse in symptoms. In relation to HEE, therapists need to be sensitive to the possibility of cultural differences in what is considered high levels of emotional expression as this may differ from one ethnic group to another (Weisman et al. 2006).

Several studies suggest that multi-family therapy group treatment is helpful as it provides an opportunity for family members to discuss common experiences and challenges (McFarlane 2002). McFarlane suggests that even when these groups are conducted at a less formal level (without therapist leadership), participants in the multi-family groups will create close relationships and networks of support for one another. Cognitive behavioral therapy (CBT) approaches have been found to be successful in treating adult-onset schizophrenia (e.g., Tarrier et al. 2004), and these treatments may hold similar promise for children if used at a developmentally appropriate level with concrete behavioral tasks that are less insight oriented and more action oriented. Utilizing reinforcers, identifying consequences, and shaping behaviors are all important components of CBT in helping the individual with schizophrenia change, but the theory also can be used to model appropriate interactions for the family members to adopt.

Marley (2004) suggests that the classic family system models still possess many important concepts that MFTs can use in the treatment of schizophrenia. The Bowenian family therapy technique of using a genogram may offer the family a broader perspective of their family supports, and help them identify other family members who may have unidentified or identified eccentricities or symptoms similar to schizophrenia, which can help demystify family secrets and shame. The stress and chaos of schizophrenia can provide a fertile environment for triangulation to occur with parents suggesting that "if only their child were cured their life would be happy." Consequently, creating a temporary coalition with the child may help detriangulate the relationship and give the child needed support. Borrowing from Whitaker's theory of the "battle for initiative," Marley proposes that the family may come to therapy expecting the therapist to "fix" their problems. Psychoeducation is an important component of the treatment of schizophrenia but in the battle for initiative the therapist should help the family become more involved in the treatment and encourage them to take the initiative in learning more. 
Structural family therapy also offers the concepts of boundaries, coalitions, detouring, and hierarchy that can all be forced to extremes under the chaotic influence of schizophrenia. Being able to bring back a healthier level of interaction using joining, boundary making, and restructuring may empower the family. While the idea of schizophrenia being caused by a double bind is no longer accepted, making such communication explicit and discussing it in relation to the family's metacommunication is a strategic method that may lead to more overt conversations that will help the family and therapist gain a better perspective of circular causality.

Therapists should strive from the beginning to create a safe place where parents and siblings of the child manifesting psychosis can express their grief, fears, and deepest emotions without being judged or blamed. MFTs who work to change the family's perceptions of the illness to a point where hope can be entertained will be of the greatest help to the family. Furthermore, it is important to increase the parents' confidence and ability to help their own child as this will reduce their perceived burden and increase their feelings of competence in parenting. This also will help reduce potential emergencies, and most importantly, give the child a greater chance of recovery so he or she can spend more time out of the hospital and in a safe, welcoming home.

\section{Conclusion}

The empirical study of childhood schizophrenia is developing and further discoveries are expected relative to the field's understanding of the brain and its relation to daily functioning. As a mental illness, schizophrenia is a pervasive and difficult disorder and can change dramatically the lives of those it directly and indirectly impacts. Isolation from loved ones is not uncommon in adults exhibiting paranoid symptomatology, but children usually are kept at home until they can support themselves and so the opportunity to practice family therapy is increased. In addition, MFTs often are affiliated with settings where they could be the first line of defense in identifying and assessing for psychosis in children (i.e., schools, churches, medical practices). Therapists who are familiar with the associated symptoms in children will be able to make an important difference in the lives of those they treat or refer for additional treatment.

Schizophrenia can be draining for families in terms of time, emotional and physical energy, and financial resources. There are a variety of treatments available for helping families struggling with schizophrenia but few are specialized and empirically supported for children and adolescents. There is a great need for therapists to be more competent in helping families acclimate to the new and sometimes overwhelming bio/psycho/social culture of schizophrenia. This article has presented some of the ways that a therapist can be more culturally competent in treating childhood schizophrenia through increased knowledge, awareness, and skill. Nevertheless, as Sue and Sue (1992) point out, a competent therapist is always actively seeking more knowledge, identifying limitations, and recognizing the complexity and diversity of the client population.

\section{References}

American Psychiatric Association (2000). Diagnostic and statistical manual of mental disorders (4th ed., text revision). Washington, DC: Author.

Armentaros, J. L., \& Davies, M. (2006). Antipsychotics in early onset schizophrenia: Systematic review and meta-analysis. European Child Adolescent Psychiatry, 15, 141-148. 
Asarnow, J. R. (1994). Childhood-onset schizophrenia. Journal of Child Psychology \& Psychiatry, 35(8), 1345-1371.

Boss, P., Caron, W., \& Horbal, J. (1988). Alzheimer's disease and ambiguous loss. In C. S. Chilam, E. W. Nunnally \& F. M. Cox (Eds.), Chronic illness and disability (pp. 123-140). Thousand Oaks, CA, US: Sage Publications, Inc.

Boss, P., \& Couden, B. (2002). Ambiguous loss from chronic physical illness: Clinical interventions with individuals, couples, and families. Journal of Clinical Psychology, 53(11), 1351-1360.

Brown, L., Macintyre, K., \& Trujillo, L. (2003). Interventions to reduce HIV/AIDS stigma: What have we learned? AIDS Education and Prevention: An Interdisciplinary Journal, 15(1), 49-69.

Butzlaff, R. L., \& Hooley, J. M. (1998). Expressed emotion and psychiatric relapse. Archives of General Psychiatry, 55(6), 547-552.

Cantor, S. (1988). Childhood schizophrenia. New York: The Guilford Press.

Chaffey, L., \& Fossey, E. (2004). Caring and daily life: Occupational experiences of women living with sons diagnosed with schizophrenia. Australian Occupational Therapy Journal, 51(4), 199-207.

Chin, S. H., \& Baton, R. (2006). Attitudes and perceptions toward depression and schizophrenia among residents in different medical specialties. Academic Psychiatry, 30(3), 262-263.

Crim, E. R., \& Fitzpatrick, J. C. (2003). A new frontier: Childhood onset mental illness. Family Therapy Magazine, 2, 34-40.

DelBello, M., \& Grcevich, S. (2004). Phenomenology and epidemiology of childhood psychiatric disorders that may necessitate treatment with atypical antipsychotics. Journal of Clinical Psychiatry, 65(Supp 16), 12-19.

Drake, R. J., Pickles, A., Bentall, R. P., Kinderman, P., Haddock, G., Tarrier, N., \& Lewis, S. W. (2004). The evolution of insight, paranoia and depression during early schizophrenia. Psychological Medicine, 34, 285-292.

Dulmus, C. N., \& Smyth, N. J. (2000). Early-onset schizophrenia: A literature review of empirically based interventions. Child \& Adolescent Social Work Journal, 17(1), 55-69.

Fagerlund, B., Pagsberg, A. K., \& Hemmingsen, R. P. (2006). Cognitive deficits and levels of IQ in adolescent onset schizophrenia and other psychotic disorders. Schizophrenia Research, 85, 30-39.

Ferriter, M., \& Huband, N. (2003). Experiences of parents with a son or daughter suffering from schizophrenia. Journal of Psychiatric and Mental Health Nursing, 10(5), 552-560.

Gartner, A. (1985). Countertransference issues in the psychotherapy of adolescents. Journal of Child \& Adolescent Psychotherapy, 2(3), 187-196.

Gerace, L. M., Camilleri, D., \& Ayres, L. (1993). Sibling perspectives on schizophrenia and the family. Schizophrenia Bulletin, 19(3), 637-647.

Getz, G. E., Fleck, D. E., \& Strakowski, S. M. (2001). Frequency and severity of religious delusions in Christian patients with psychosis. Psychiatry Research, 103(1), 87-91.

Gilligan, J., \& Lee, B. (2004). The psychopharmacologic treatment of violent youth. In J. G. Devine, J. Gilligan, K. A. Miczek, R. Shaikh \& D. Pfaff (Eds.), Youth violence: Scientific approaches to prevention (pp. 356-381). New York: New York Academy of Science.

Gogtay, N., Sporn, A., Clasen, L. S., Nugent, T. F., Greenstein, D., Nicolson, R., et al. (2004). Comparison of progressive cortical gray matter loss in childhood-onset schizophrenia with that in childhood-onset atypical psychoses. Archives of General Psychiatry, 61(1), 17-22.

Gur, R. E., \& Johnson, A. B. (2006). If your adolescent has schizophrenia: An essential resource for parents. New York: Oxford University Press.

Hafner, H., \& Nowotny, B. (1995). Epidemiology of early-onset schizophrenia. European Archives of Psychiatry and Clinical Neuroscience, 245(2), 80-92.

Hartshorne, T. S. (2002). Mistaking courage for denial: Family resilience after the birth of a child with severe disabilities. The Journal of Individual Psychology, 58(3), 263-278.

Hata, K., Iida, J., Iwasaka, H., Negoro, H., Ueda, F., \& Kishimoto, T. (2003). Minor physical anomalies in childhood and adolescent onset schizophrenia. Psychiatry and Clinical Neurosciences, 57(1), 17-21.

Kuipers, E. (2006). Family interventions in schizophrenia: Evidence for efficacy and proposed mechanisms of change. Journal of Family Therapy, 28(1), 73-80.

Lev, E. L., \& McCorkle, R. (1998). Loss, grief, and bereavement in family members of cancer patients. Seminars in Oncology Nursing, 14(2), 145-151.

Lewis, S. W., Tarrier, N., \& Drake, R. J. (2005). Integrating non-drug treatments in early schizophrenia. British Journal of Psychiatry, 187(Suppl 48), s65-s71.

Marley, J. A. (2004). Family involvement in treating schizophrenia: Models, essential skills, and process. New York: The Haworth Press.

Marsh, D. T., \& Johnson, D. L. (1997). The family experience of mental illness: Implications for intervention. Professional Psychology: Research and Practice, 28(3), 229-237. 
Maziade, M., Gingras, N., Rodrigue, C., Bouchard, S., Cardinal, A., Gauthier, B., et al. (1996). Long-term stability of diagnosis and symptom dimensions in a systemic sample of patients with onset of schizophrenia in childhood and early adolescence. I: Nosology, sex and age of onset. British Journal of Psychiatry, 169, 361-370.

McClellan, J. M., Werry, J. S., \& Ham, M. (1993). A follow-up study of early onset psychosis: Comparison between outcome diagnoses of schizophrenia, mood disorders, and personality disorders. Journal of Autism and Developmental Disorders, 23(2), 243-262.

McFarlane, W. R. (2002). Multifamily groups in the treatment of severe psychiatric disorders. New York: The Guilford Press.

Morris, D. B. (2000). Illness and culture in the postmodern age (Vol. 1). University of California Press.

Mueser, K. T., Crocker, A. G., Frisman, L. B., Drake, R. E., Covell, N. H., \& Essock, S. M. (2006). Conduct disorder and antisocial personality disorder in persons with severe psychiatric and substance use disorders. Schizophrenia Bulletin, 32(4), 626-636.

Ng, R. M. K., Mui, J., Cheung, H. K., \& Leung, S. P. (2001). Expressed emotion and relapse of schizophrenia in Hong Kong. Hong Kong Journal of Psychiatry, 11(1), 4-11.

Northey, W. F., \& Harrington, M. (2003). 2002 AAMFT member survey of clinical practices and approaches to substance abuse. Alexandria, VA: American Association of Marriage and Family Therapy.

Patterson, J. M. (2002). Understanding family resilience. Journal of Clinical Psychology, 58(3), $233-246$.

Pompili, M., Ruberto, A., Girardi, P., \& Tatarelli, R. (2004). Understanding suicide in schizophrenia: A national imperative for prevention. Psichiatria e Psicoterapia, 23(4), 275-281.

Prince, J. D. (2006). Practices preventing rehospitalization of individuals with schizophrenia. Journal of Nervous and Mental Disease, 19(6), 397-403.

Russell, A. T., Bott, L., \& Sammons, C. (1989). The phenomenology of schizophrenia occurring in childhood. Journal of the American Academy of Child and Adolescent Psychiatry, 28, 399-407.

Schwartz-Stav, O., Apter, A., \& Zalsman, G. (2006). Depression, suicidal behavior and insight in adolescents with schizophrenia. European Child \& Adolescent Psychiatry, 15(6), 352-359.

Shaw, M., \& Singh, S. P. (2004). Management of early-onset psychosis. Current Opinion in Psychiatry, 17(4), 249-254.

Sowell, E. R., Toga, A. W., \& Asarnow, R. (2000). Brain abnormalities observed in childhood-onset schizophrenia: A review of the structural magnetic resonance imaging literature. Mental Retardation and Developmental Disabilities Research Reviews, 6(3), 180-185.

Sue, D. W., Arredondo, P., \& McDavis, R. J. (1992). Multicultural counseling competencies and standards: A call to the profession. Journal of Multicultural Counseling and Development, 20, 64-88.

Sue, D. W., \& Sue, D. (2003). Counseling the culturally diverse: Theory and practice. New York: Wiley.

Tarrier, N., Lewis, S., Haddock, G., Drake, R., Kinderman, P., Kingdon, R., et al. (2004). Cognitivebehavioural therapy in first-episode and early schizophrenia. British Journal of Psychiatry, 184, 238239.

Topor, A., Borg, M., Mezzina, R., Sells, D., Marin, I., \& Davidson, L. (2006). Others: The role of family, friends, and professionals in the recovery process. American Journal of Psychiatric Rehabilitation, 9(1), 17-37.

Veen, N. D., Selten, J. P., van der Tweel, I., Feller, W. G., Hoek, H. W., \& Kahn, R. S. (2004). Cannabis use and age at onset of schizophrenia. American Journal of Psychiatry, 161(3), 501-506.

Weisman, A. G., Rosales, G. A., \& Kymalainen, J. A. (2006). Ethnicity, expressed emotion, and schizophrenia patients' perceptions of their family members' criticism. Journal of Nervous and Mental Disease, 194(9), 644-649.

Werry, J. S. (1996). Childhood schizophrenia. In F. R. E. Volkmar (Ed.), Psychoses and pervasive developmental disorders in childhood and adolescence (pp. 1-48). Washington, DC: American Psychiatric Association.

Wudarsky, M., Lenane, M., \& Rapoport, J. L. (2001). Childhood- and adolescent-onset schizophrenia. In R. M. Murray (Ed.), Comprehensive care of schizophrenia: A textbook of clinical management. Malden, MA: Blackwell Science, Inc.

Yang, P. C., Liu, C. Y., Chiang, S. Q., Chen, J. Y., \& Lin, T. S. (1995). Comparison of adult manifestations of schizophrenia with onset before and after 15 years of age. Acta Psychiatrica Scandinavica, 91, 209212. 